\title{
A Transparent Display with Per-Pixel Color and Opacity Control
}

\author{
TJ Rhodes \\ Gavin Miller \\ Adobe Research
}

\author{
Qi Sun \\ Daichi Ito \\ Adobe Research
}

\author{
Li-Yi Wei \\ Adobe Research
}

\begin{abstract}
We propose a new display system that composites matted foreground animated graphics and video, with per-pixel controllable emitted color and transparency, over real-world dynamic objects seen through a transparent display. Multiple users can participate simultaneously without any glasses, trackers, or additional devices. The current prototype is deployed as a desktop-monitor-sized transparent display box assembled from commodity hardware components with the addition of a high-frame-rate controllable diffuser.
\end{abstract}

\section{CCS CONCEPTS}

- Human-centered computing $\rightarrow$ Mixed / augmented reality; Displays and imagers; • Computing methodologies $\rightarrow$ Mixed / augmented reality.

\section{KEYWORDS}

display, perception, augmented reality

\section{ACM Reference Format:}

TJ Rhodes, Gavin Miller, Qi Sun, Daichi Ito, and Li-Yi Wei. 2019. A Transparent Display with Per-Pixel Color and Opacity Control. In Proceedings of SIGGRAPH '19 Emerging Technologies. ACM, New York, NY, USA, 2 pages. https://doi.org/10.1145/3305367.3327984

\section{INTRODUCTION}

Transparent displays can be useful to display graphical information in the presence of real-world objects, such as behind the display. Common transparent displays take two basic forms - emissive displays and transmissive displays. Transparent emissive displays emit light in all directions from each pixel. Light may also travel directly through the display, but may be attenuated by a fixed amount due to the fixed opacity of the display. This can be achieved with transparent Organic Light Emitting Diodes (OLEDs) or using a switchable diffuser with back projection [Izadi et al. 2008]. Transparent transmissive displays control the intensity of light passing through each pixel over a range of angles, but do not emit light. Liquid crystal displays (LCDs) are a common form of transmissive display.

An ideal transparent display would modulate the emissive color and transparency of each pixel allowing for the Over operator of Porter Duff compositing [Porter and Duff 1984] with real-world objects. The color image would be the graphic color with premultiplied alpha, and the transparency image would contain the color transparency mask of the graphics. One approach for a practical device would layer an OLED on top of an LCD. However, this would require an exact match of pixel pitch, careful alignment of

SIGGRAPH '19 Emerging Technologies, fuly 28 - August 01, 2019, Los Angeles, CA, USA (c) 2019 Copyright held by the owner/author(s). Publication rights licensed to ACM. This is the author's version of the work. It is posted here for your personal use. Not for redistribution. The definitive Version of Record was published in Proceedings of SIGGRAPH '19 Emerging Technologies, https://doi.org/10.1145/3305367.3327984.
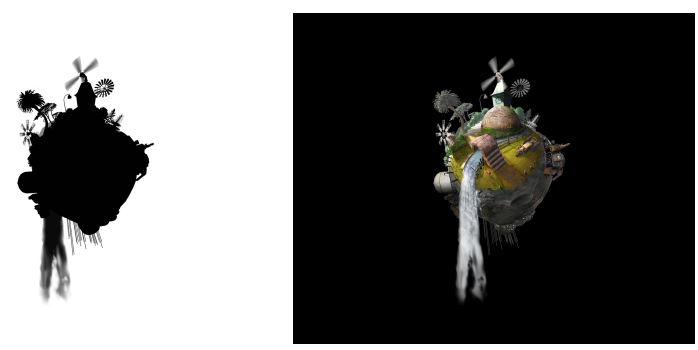

(a) transparency image

(b) color image

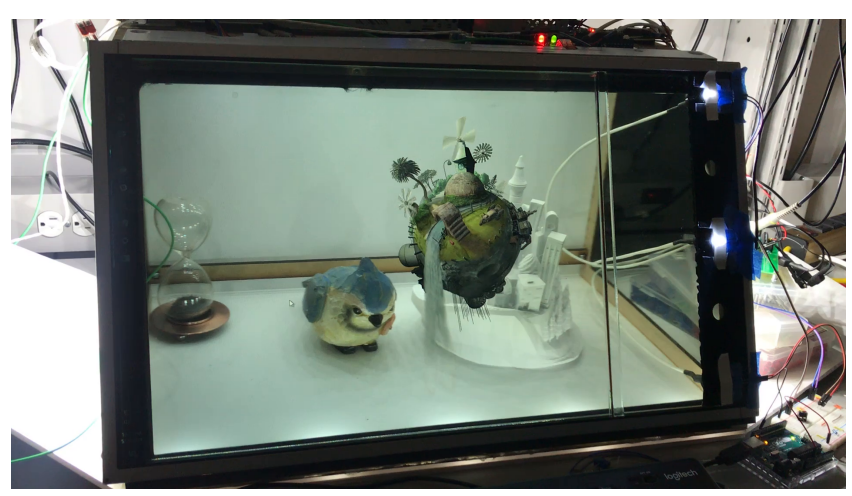

(c) two-phase composite

Figure 1: Our composite display system. The transparency image, (a), displays white to represent transparent areas while black heavily attenuates the view. The color image, (b), displays the color channel over the illuminated diffuser. At 144 Hz, the two-phase composite, (c), occludes physical objects within the display box.

the panels and may lead to viewing-direction-dependent artifacts as the two pixel structures blocked each other, leading to Moiré fringes.

Instead, we combine a conventional LCD and a switchable singleelement diffuser. The display works by time-multiplexing a transparent phase and an emissive phase, with retinal fusion leading to the perception of compositing the foreground graphic over the background real-world scene. Multiple users can simultaneously view and interact with our prototype system without any glasses, trackers, or additional devices.

\section{DESIGN}

Our composite display system consists of: an enclosure, display layer, and controller. Figure 1 illustrates our system design.

A modern LCD desktop monitor consists of a display and backlight assembly that fit within a $1 \mathrm{~cm}$ thick enclosure. We expand 
this distance enough to place physical objects between the display and backlight. Our display system box measures approximately $55 \times 30 \times 35$ (width, height, depth in $\mathrm{cm}$ ). The display face tilts into the volume of the box at a 65 degree incline. The left, right, and rear sides are equipped with hinged walls for access to insert or remove objects within the box. The backlight system uses thirty (3-up) high-power LED stars. The LEDs are divided amongst eight LED rails with separate drivers. Depending on the configuration, a portion of the LED rails illuminate the placed objects while the remainder illuminate the display layer. The backlight system is capable of producing 13, $740 \mathrm{~lm}$ (luminous flux) combined.

The display layer consists of an LCD stacked on top of a switchable diffuser. The layer is oriented so the diffuser faces the interior and the LCD faces the viewer. The LCD is harvested from an ordinary NVIDIA 3D Vision ready consumer-grade desktop monitor. The display layer shown in Figure 1 uses a $1920 \times 1080$, 24.5", 240 $\mathrm{Hz}$, twisted-nematic (TN) display. However, our system has been tested to work with other LCDs at various resolutions and refresh rates. The single-element switchable diffuser alternates between a scattering (diffuse) and transparent state.

The controller consists of: a microcontroller, protective circuitry, and individual LED rail drivers. Together, they synchronize the display layer components and lights to produce the desired effect. The microcontroller uses pulse width modulation (PWM) dimming to control the backlight system. The switchable diffuser is controlled through protective circuitry to ensure it does not remain energized. The LCD is driven by the original video controller harvested from the desktop monitor, and an ordinary frame buffer located in a separate host computer. DVI or DisplayPort is required to display content due to the high frame rate.

\section{IMPLEMENTATION}

Figure 2 exemplifies our digital overlay with variable opacity over physical objects. We rapidly alternate between a transparency phase and color phase to achieve this result.

The transparency phase is responsible for per-pixel attenuation of the enclosure interior. An LCD modulates the light traveling through it over a range of angles. We leverage this property by filling the frame buffer with a per-color-channel transparency mask. White pixels represent transparent areas while black pixels heavily attenuate the view behind the display layer (down to the contrast ratio of the display). Intermediate transparency values attenuate the light proportionately. The switchable diffuser is transparent during this phase, so many view directions through the display are appropriately modulated by the transparency image.

The color phase is responsible for displaying the color channel of the desired content. The switchable diffuser is scattering during this phase, so nothing within the enclosure interior is visible. The white backlight system illuminates the diffuser, making the display layer function as an ordinary LCD assembly. The frame buffer contains the per channel color pre-multiplied by one minus per-channel transparency for each pixel.

The two-phase composite is generated by alternating between the transparency and color phases at 144 frames per second (fps). This frame rate enables us to display not only static images, but

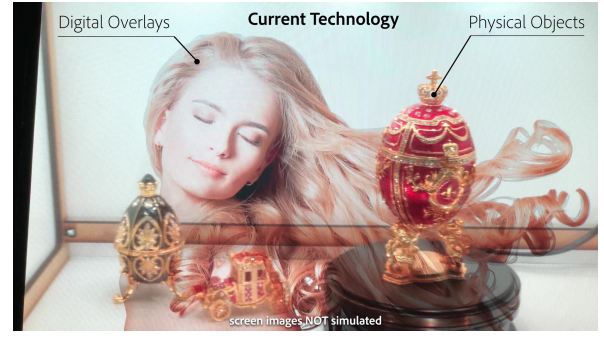

(a) half opacity

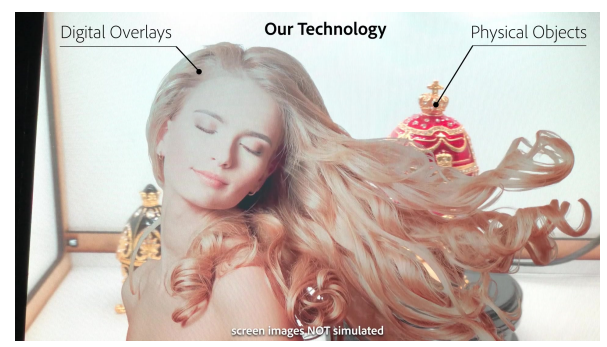

(b) full opacity

Figure 2: Per-pixel opacity control.

also dynamic animations and video at $72 \mathrm{fps}$. The diffuser is synchronized with the frame buffer using a VESA stereo signal from the host computer.

\section{DISCUSSION}

The progressive display of scanlines on the LCD combined with a single-element diffuser leads to cross-talk artifacts between the transparency and color phases. We address this by flashing the backlight briefly for each phase, after the full frame is displayed.

The two-phase composite is displayed in a single plane in front of the real-world objects inside the box. The result is view-dependent based on the LCD's viewing angle characteristics. Additionally, we reduce the frame rate from its theoretical maximum of $240 \mathrm{fps}$ to $144 \mathrm{fps}$ due to the real-world rate at which pixels can switch from white to black. The current display frame rate prevents the use of shutter glasses for frame-sequential stereo display.

As LCD technology advances, the two-phase composite technique will scale for larger and faster displays. Specifically, a faster frame rate panel will enable experimentation with frame-sequential stereo display - with the addition of shutter glasses.

\section{REFERENCES}

Shahram Izadi, Steve Hodges, Stuart Taylor, Dan Rosenfeld, Nicolas Villar, Alex Butler, and Jonathan Westhues. 2008. Going Beyond the Display: A Surface Technology with an Electronically Switchable Diffuser. In UIST '08. 269-278. https://doi.org/ $10.1145 / 1449715.1449760$

Thomas Porter and Tom Duff. 1984. Compositing Digital Images. In SIGGRAPH '84. 253-259. https://doi.org/10.1145/800031.808606 